Revue d'histoire de l'Amérique française

RAS REVUE D.HISTOIRE DE L'AMÉRIQUE FRANÇAISE

\title{
Religion, science et autorité dans le développement des sciences sociales en Amérique du Nord
}

\section{Marlene Shore}

Volume 57, numéro 3, hiver 2004

URI : https://id.erudit.org/iderudit/009594ar

DOI : https://doi.org/10.7202/009594ar

Aller au sommaire du numéro

Éditeur(s)

Institut d'histoire de l'Amérique française

\section{ISSN}

0035-2357 (imprimé)

1492-1383 (numérique)

Découvrir la revue

\section{Citer cet article}

Shore, M. (2004). Religion, science et autorité dans le développement des sciences sociales en Amérique du Nord. Revue d'histoire de l'Amérique française, 57(3), 371-388. https://doi.org/10.7202/009594ar d'utilisation que vous pouvez consulter en ligne. 


\title{
Religion, science et autorité dans le développement des sciences sociales en Amérique du Nord
}

MARLENE SHORE

Département d'histoire

Université York

\begin{abstract}
A UNE ÉPOQUE où la formation générale est incomprise du public et fait l'objet de critiques des gouvernements pour sa supposée inutilité, il importe de rappeler la dynamique qui a présidé au développement des sciences sociales dans les universités nord-américaines. Ces sciences se sont développées, puis ont été introduites dans les institutions de haut savoir nord-américaines dans la seconde moitié du $\mathrm{xIx}^{\mathrm{e}}$ siècle où elles se sont plus solidement implantées au début des années 1920. Dans la foulée de la Première Guerre mondiale, on avait généralement acquis la conviction que les universités devraient être davantage à l'écoute des communautés qui les entouraient. En proposant des interprétations du comportement humain, de la société et de l'économie, les sciences sociales ouvraient la perspective d'un tel rapprochement. Parce qu'elles semblaient aussi épouser les préoccupations de la grande entreprise de cette époque - accroître l'efficience industrielle - ces disciplines retinrent l'attention d'administrateurs d'universités de plus en plus dépendantes de bienfaiteurs privés pour leur financement. Durant l'entre-deux-guerres, néanmoins, les sciences sociales furent considérées avec méfiance, car on les supposait intrinsèquement porteuses de critique sociale. Dans plusieurs universités nord-américaines, dont McGill à Montréal, les professeurs de sciences sociales devinrent la cible d'administrateurs désireux de débarrasser leur institution de ce qu'on percevait comme des foyers de radicalisme.
\end{abstract}


Cette méfiance à l'endroit des sciences sociales était paradoxale, surtout si l'on se rappelle que leurs origines sont religieuses et qu'elles sont nées de la philosophie morale. Elles se trouvèrent pourtant plongées au cœur de controverses car, même issues de traditions et de disciplines humanistes, elles s'étaient aussi développées en réponse à des conditions sociales difficiles et à une évolution scientifique qui remettaient en question la nature et le siège de l'autorité. Tel est le thème que nous abordons dans cette étude, sans toutefois postuler l'existence d'une dichotomie entre science et religion dans la construction des sciences sociales; nous poserons plutôt l'existence, au cœur même des sciences humaines, d'une dualité — c'est-à-dire d'une position qui donne à penser que, parfois, elles visent au maintien du statu quo et que, d'autres fois, elles mettent en péril institutions et traditions établies.

Aux États-Unis, la psychologie, l'anthropologie, l'économie, la sociologie et la science politique ne commencèrent à émerger en tant qu'entités intellectuelles distinctes que dans les années 1870 et 1880 . Chacune relevait d'une tradition de pensée, mais elles n'intéressaient surtout que les personnes engagées à proposer des solutions à des problèmes concrets auprès des gouvernements ou dans le monde de l'éducation, ou encore des universitaires et des membres du clergé qui aimaient discuter d'histoire, de philosophie morale et de théologie naturelle — cette large tradition humaniste d'où étaient issues les sciences sociales.

À compter du début du XIX ${ }^{\mathrm{e}}$ siècle, à la fois aux États-Unis et au Canada, les cours de morale et de philosophie de l'esprit (c'est-à-dire à la fois psychologie, logique et métaphysique) constituèrent le couronnement du cursus collégial. Les collèges étaient tous dominés par des considérations religieuses et, dans les cours de morale et de philosophie de l'esprit, les divers aspects de la vie sociale étaient étudiés en tant que subdivisions du comportement moral dans un monde naturel gouverné par Dieu. Les premiers collèges américains et anglo-canadiens (ou de l'Amérique du Nord britannique) furent institués pour former une élite sociale et morale; on y considérait ces cours de philosophie comme essentiels à l'apprentissage et au maintien des valeurs propres à l'autorité morale protestante. Au Canada, la morale philosophique allait demeurer inchangée jusqu’à la fin $\mathrm{du} \mathrm{xIx}^{\mathrm{e}}$ siècle et exercer longtemps, au xx ${ }^{\mathrm{e}}$, une forte influence sur la nature de l'éducation supérieure canadienne ${ }^{1}$.

1. Dorothy Ross, "The Development of the Social Sciences», dans Alexandra Oleson et John Voss, dir., The Organization of Knowledge in Modern America (Baltimore/London, The Johns Hopkins University Press, 1979), 107-109; A. B. McKillop, A Disciplined Intelligence. Critical Inquiry and Canadian Thought in the Victorian Era (Montreal, McGill-Queen's University Press, 1980), 28-33, 58, 
Aux États-Unis, la morale et la philosophie de l'esprit se scindèrent en plusieurs disciplines distinctes durant les années 1870 et 1880 : toutes s'établirent dans les institutions universitaires et mirent l'accent sur la spécialisation. Quatre des cinq sciences sociales formèrent leur premier département d'études supérieures et créèrent leur revue professionnelle durant les années 1880; la sociologie en fit autant dans les années 1890. Ce faisant, les nouvelles sciences sociales suivaient une tendance amorcée dans d'autres disciplines. C'est ainsi, par exemple, que les sciences naturelles s'étaient organisées professionnellement, avant la Guerre de Sécession, en créant l'American Association for the Advancement of Science; après la guerre, disposant de nouvelles bases dans les universités, elles formèrent des organisations professionnelles distinctes. Philologues et historiens entendaient aussi spécialiser et professionnaliser leur discipline : avant même que l'une quelconque des sciences sociales se soit dotée d'une organisation, l'American Philological Society était mise sur pied en 1869 et la Modern Language Association, en $1883^{2}$.

Des pressions s'exerçaient aussi pour que les disciplines universitaires deviennent plus scientifiques, afin de les amener à concorder avec les aspirations professionnelles et scientifiques d'autres secteurs d'activité ${ }^{3}$. Les décennies 1870 et 1880 furent une époque de dépression économique, de conflits ouvriers et d'agitation sociale entraînés par une rapide croissance urbaine et industrielle. Ces problèmes provoquèrent une crise chez les intellectuels. Comme plusieurs de leurs contemporains actifs dans d'autres domaines, les premiers praticiens des sciences sociales étaient généralement issus de familles protestantes nées aux États-Unis et se considéraient comme membres de l'élite de la société. Désireux d'exercer leur leadership mais redoutant, en même temps, la menace que les problèmes sociaux faisaient peser sur leur propre situation, ils furent de plus en plus nombreux à s'intéresser aux réformes sociales, aux organisations caritatives, à la réforme de la fonction publique, de l'éducation et du système économique.

Lorsque les collèges, soumis aux mêmes pressions, passèrent au régime électif ${ }^{4}$ et introduisirent un cursus susceptible de plaire davantage à leur

95-99; Laurence Veysey, The Emergence of the American University (Chicago, University of Chicago Press, 1965), 23-31, 60.

2. Dorothy Ross, loc. cit., 108.

3. Ibid., 109; voir aussi Burton Bledstein, The Culture of Professionalism. The Middle Class and the Development of Higher Education in America (New York, Norton, 1976); Thomas Haskell, The Emergence of Professional Social Science (Urbana, University of Illinois Press, 1977).

4. Le régime autorise les étudiants à choisir leurs cours plutôt que d'être astreints à un programme imposé. 
clientèle de classe moyenne, à leurs administrateurs et à leurs anciens élèves, ces praticiens introduisirent des cours sur les problèmes sociaux — c'est-à-dire la "pathologie sociale » — dans lesquels on accompagnait les étudiants au cour des villes pour y observer ceux qu'on qualifiait de "délinquants», "anormaux» et "dépendants». De tels cours, axés sur la résolution de problèmes concrets, sont encore un des traits marquants de la sociologie ${ }^{5}$. L'influence de la tradition évangéliste protestante, dans laquelle avaient grandi la plupart des premiers praticiens, les porta aussi à s'intéresser à l'amélioration morale.

La concurrence venue de prétendants populaires à la science et à l'utilité sociale fut un autre facteur déterminant dans l'évolution des sciences sociales. Ainsi, par exemple, des psychologues éprouvèrent le besoin de s'opposer aux spiritualistes et aux chercheurs en parapsychologie, et les sociologues luttèrent contre le clergé et les réformateurs laïques qui prônaient des réformes plus radicales que celles qu'ils étaient disposés à sanctionner ${ }^{6}$.

On peut certes voir dans l'agitation sociale la raison de l'intérêt que portèrent aux enquêtes sociales ces réformateurs et ces universitaires nordaméricains de la fin du XIX ${ }^{\mathrm{e}}$ siècle, mais cela n'explique pas ce qui les poussa à donner à ces travaux un caractère de plus en plus scientifique. En fait, cette tendance reflétait l'augmentation des connaissances qui, dans chaque domaine, s'était produite en Europe au siècle précédent. Dans chacune des sciences sociales, les universitaires américains des années 1870 s'ouvrirent peu à peu aux théories et aux pratiques développées outre-mer. À l'exception de l'économie politique classique, toutes les disciplines repensèrent leurs cadres théoriques en fonction des hypothèses de l'évolutionnisme biologique : en anthropologie, on formulait une théorie universelle de l'évolution; en sociologie, Comte et Spencer proposaient leurs théories positivistes de l'évolution sociale et, en science politique, on avançait des théories de l'évolution historique des institutions politiques anglo-américaines.

Dans les années 1880, la formation d'une nouvelle génération de scientifiques américains dans des universités allemandes (ou dans des institutions de recherche américaines, telle Johns Hopkins) accéléra cette tendance. À cette époque, la Wissenschaft allemande atteignait le point culminant de sa phase empirique et, soit parce qu'ils y avaient été exposés, soit en raison de l'influence profonde de la théorie darwinienne sur la biologie, les chercheurs

5. Mary Furner, Advocacy and Objectivity (Lexington, University of Kentucky Press, 1975).

6. Dorothy Ross, loc. cit., 111-112 ; J. C. Burnham, How Superstition Won and Science Lost. Popularizing Science and Health in the United States (New Brunswick, N. J., Rutgers University Press, 1987), 9, 34, 41, 86. 
de la jeune génération en sciences sociales en conclurent que la "véritable» science n'édifiait ses théories qu'en suivant de très près la recherche empirique. Ce que cela signifiait demeurait assez vague : ils entretenaient des conceptions diverses de ce qui constituait la science; en outre, dans toutes les sciences sociales, de nombreux universitaires fondaient leur approche empirique sur une nouvelle conscience historique. En d'autres termes, la forte influence de l'historicisme allemand sur les sciences sociales des années 1880 mena à définir la méthode scientifique en relation avec l'histoire tout autant qu'avec les sciences sociales ${ }^{7}$.

La psychologie fournit un exemple particulièrement significatif de la manière dont les préoccupations concernant la nature et le siège de l'autorité, les élans de réforme sociale et l'adoption d'une méthode scientifique se développèrent parallèlement à l'émergence d'une conscience historique pour façonner les sciences sociales. Désignée sous ce nom pour la première fois au $\mathrm{XvIII}^{\mathrm{e}}$ siècle, la psychologie était une branche de la philosophie traitant de la conscience et de la vie de l'esprit. Jusqu'à la fin du $\mathrm{XIX}^{\mathrm{e}}$ siècle, l'étude de la conscience procédait essentiellement par introspection, mais ce mode s'effaça peu à peu devant les efforts pour établir la psychologie comme une science objective de l'expérience subjective. Au début des années 1890, la psychologie s'engageait dans la voie, empruntée à la physiologie, de la recherche expérimentale en laboratoire. Cette nouvelle orientation venait d'Allemagne, où Wilhelm Wundt avait créé en 1879 le premier laboratoire de psychologie, exemple bientôt suivi dans d'autres villes européennes et nord-américaines. En Amérique du Nord, c'est essentiellement par ses recherches en laboratoire que la psychologie parvint à s'imposer comme discipline autonome - et à se détacher ainsi de la philosophie ${ }^{8}$. Au Canada, la psychologie expérimentale fut très tôt pratiquée : en 1889, l'université de Toronto accorda une chaire de philosophie à l’Américain James Mark Baldwin, psychologue expérimental formé par

7. Dorothy Ross, loc. cit., 111-114; Jurgen Herbst, The German Historical School in American Scholarship (Ithaca, Cornell University Press, 1969), 295-315.

8. John M. O’Donnell, Origins of American Behaviorism (New York/London, New York University Press, 1983), 1, 7, 16, 225 ; John C. Burnham, "The New Psychology», dans Adele Heller et Lois Rudnick, dir., 1915. The Cultural Moment (New Jersey, Rutgers University Press, 1991), 118 ; John C. Burnham, "How Superstition Won and Science Lost ", 87-88; L. S. Hearnshaw, The Shaping of Modern Psychology : An Historical Introduction (London/New York, Routledge, 1987); nouvelle édition 1989, 134-138; Michael M. Sokal, "Origins of the American Psychological Association", dans Rand B. Evans, Virginia Staudt Sexton et Thomas C. Cadwallader, dir., 100 Years. The American Psychological Association : A Historical Perspective (Washington, American Psychological Association, 1992), 46. 
Wilhelm Wundt, non sans que cette nomination provoque une avalanche de protestations. Les collègues désignés comme "nativistes» souhaitaient voir le poste accordé à James Gibson Hume, un Canadien. Afin de calmer le jeu, le département segmenta la chaire de philosophie en deux parties : Baldwin devint professeur de logique, de métaphysique et d'éthique, tandis que Hume fut nommé professeur d'histoire de la philosophie. Baldwin croyait que l'opposition à sa nomination venait de ce qu'on le considérait comme un "matérialiste». Cependant, Toronto se hâta d'introduire un cours de psychologie expérimentale et Hume devint, de même que Baldwin, membre de la nouvelle Association américaine consacrée à l'avancement de la psychologie en tant que discipline scientifique ${ }^{9}$.

Pendant que la psychologie, en s'orientant vers l'expérimentation en laboratoire, conquérait progressivement son autonomie en Amérique du Nord, on lui accorda une reconnaissance officielle à la World Columbian Exposition tenue à Chicago en 1893 - qui marquait sa première présence à une exposition internationale ${ }^{10}$. Les principaux psychologues nord-américains, dont Balwin, y virent une occasion idéale de présenter leur travail à un plus vaste public, espérant ainsi être mieux reconnus comme professionnels et contrer la superstition, le faux mysticisme et autres formes de ce qu'ils considéraient comme des pseudo-sciences pour lesquelles l'intérêt populaire ne se démentait pas.

Ce virage scientifique de la psychologie, que manifestait en partie, à la fin du $\mathrm{XIX}^{\mathrm{e}}$ siècle, le recours à la méthode expérimentale, en rendait la démonstration publique plus facile qu'à l'époque où elle était liée à la philosophie, et la nouvelle discipline mérita ainsi un soutien populaire et institutionnel. Mais le prestige de plus en plus grand - et l'autorité - de la science en Occident n'expliquent pas à eux seuls la nouvelle orientation expérimentale de la psychologie à la fin $\mathrm{du} \mathrm{xIx}^{\mathrm{e}}$ siècle. Ce tournant est aussi attribuable à la prééminence du visuel dans la culture occidentale, dont un des traits les plus marqués a été de réifier la pensée, d'extérioriser et d'objectiver les états intérieurs ${ }^{11}$. À cet élément formateur de la psychologie s'ajoutèrent, à la

9. James Mark Baldwin, Between Two Wars, 1861-1921 (Boston, The Stratford Co., 1926), I : 41; Mary Wright et C. Roger Myers, dir., History of Academic Psychology in Canada (Toronto, C. J. Hogrefe Inc., 1982), 70-73.

10. Marlene Shore, «Psychology and Memory in the Midst of Change: The Social Concerns of Late-19th-Century North American Psychologists ", dans Christopher Green, Marlene Shore et Thomas Teo, dir., The Transformation of Psychology. Influences of $19^{\text {th }}$-Century Philosophy, Technology, and Natural Science (Washington, American Psychological Association, 2002), 63-86.

11. Elaine Scarry, The Body in Pain: The Making and Unmaking of the World (New York, Oxford University Press, 1985), 4-5, 13-22, 79. 
fin du $\mathrm{XIx}^{\mathrm{e}}$ siècle, les travaux théoriques sur la vision elle-même, particulièrement sur la confiance accordée à la vision — à ce que l'œil perçoit dans une période de rapides changements économiques, technologiques et sociaux, alors que disparaissait une grande part de l'environnement familier. Corrélativement, les psychologues commencèrent alors à s'intéresser aux recherches sur la mémoire à une époque où d'autres s'inquiétaient de l'oblitération du passé.

À la fin du XIx ${ }^{\mathrm{e}}$ siècle, il était généralement admis que les changements rapides causés par l'urbanisation et l'industrialisation avaient mis en péril le rapport de la société à son passé. Bien que d'autres époques aient connu une telle tension interne, plusieurs historiens ont noté que la période allant du XviII siècle aux années 1920 - ce qu’on a appelé "le long xix ${ }^{\mathrm{e}}$ siècle» — fut particulièrement traumatisante. L'introduction de la machine, qui fit faire un bond à la productivité humaine et facilita la multiplication et la dissémination des biens d'utilité courante, provoqua de nombreuses réflexions sur l'impact de la technologie et sur les systèmes économiques et sociaux qui en dépendaient de plus en plus. Il en résulta, notamment, l'émergence de ce que l'École de Francfort a qualifié d' «industrie de la conscience», c'est-à-dire l'ensemble des activités ayant trait à l'institutionnalisation de la mémoire culturelle, telles certaines cérémonies politiques nationales (comme les expositions mondiales) et l'édification de monuments. Participe de la même mentalité la multiplication des efforts pour conserver le passé sous la forme d'archives - par la collecte et l'entreposage de documents écrits — afin qu'il ne survive plus seulement dans la mémoire des vivants mais soit sauvegardé par d'autres mécanismes et dans d'autres institutions. La fin du xix ${ }^{e}$ siècle fut marquée par la création et la prolifération de musées, gardiens des artéfacts de sociétés anciennes, et de galeries d'art où l'on proposait des images dont le symbolisme était porteur de souvenirs culturels. Ce désir de recouvrer et de sauvegarder le passé se manifesta aussi par l'émergence de l'anthropologie, qui étudie les systèmes de croyances incarnés dans les danses rituelles, les cérémonies religieuses, les chants et les mythes, de même que par un intérêt parallèle pour le "primitif» et l'« exotique $^{12}$ ».

L'histoire elle-même, qui s'en étonnerait, devint un mode d'enquête privilégié à la fin du $\mathrm{XIX}^{\mathrm{e}}$ siècle et se donna rapidement des cadres disciplinaires

12. Patricia Fara et Karalyn Patterson, «Introduction», dans Patricia Fara et Karalyn Patterson, dir., Memory (Cambridge, Cambridge University Press, 1998), 3 ; Matt K. Matsuda, The Memory of the Modern (New York, Oxford University Press, 1996), 12; voir aussi Richard Terdiman, Present Past. Modernity and the Memory Crisis (Ithaca, Cornell University Press, 1993). 
professionnels partout en Europe et en Amérique. Alors que la philosophie avait revendiqué le titre de reine des disciplines au xviII ${ }^{\mathrm{e}}$ siècle, l'histoire avait entrepris de la remplacer dès le milieu du xix ${ }^{e}$. Tableaux et romans historiques gagnèrent en faveur; dans le champ des arts, l'étude et la critique se transformèrent en histoire de l'art ou de la littérature. L'architecture urbaine (gares ferroviaires, banques, édifices parlementaires, hôtels de ville) emprunta les styles d'époques antérieures. Comme l'a soutenu Carl Schorske, l'historicisme s'imposa à la culture comme un moyen d'apprivoiser la modernisation en rassemblant les ressources du passé dans une ère d'ascension du nationalisme, alors que les identités collectives étaient en voie de redéfinition.

À la fin du $\mathrm{XIX}^{\mathrm{e}}$ siècle, une réaction survint sous la forme du modernisme, alors qu'intellectuels et artistes de divers horizons s'efforcèrent de confronter la modernité dans ses propres termes. Mais c'était là l'envers de la même médaille. Comme l'a observé Schorske : «maitriser la modernité en pensant avec l'histoire, maîtriser la modernité en pensant sans l'histoire, ce ne sont pas là de simples antithèses mais les étapes successives de la même volonté de donner forme et sens à la civilisation européenne à l'ère du capitalisme industriel et de la montée de la démocratie politique ${ }^{13}$."

Le même contexte contradictoire donna naissance, à la même époque, à une quasi-obsession pour le phénomène de la mémoire. Dans de nombreux milieux culturels et dans diverses disciplines, la mémoire devint un objet d'études et l'on mit au point diverses techniques pour en explorer le fonctionnement. Ce faisant, cependant, les physiologistes de la fin du XIX ${ }^{\mathrm{e}}$ siècle ne se donnaient pas de leur objet une définition particulière. Ils partageaient plutôt, semble-t-il, la fascination ambiante pour ses multiples sens et connotations; "mémoire" pouvait ainsi renvoyer à la capacité de se souvenir du passé, manifestant ainsi une fonction généralement attribuée au cerveau, ou connoter quelque chose de plus abstrait - le souvenir d'une personne, d'un sentiment ou d'un événement passé. Bien entendu, ces deux notions étaient étroitement interconnectées. Selon un historien de la culture européenne de la fin du XIx ${ }^{\mathrm{e}}$ siècle, cette époque a conçu la mémoire comme "une manière de concevoir globalement faits vécus et observations dans le langage de la disparition et du changement, de la fugacité, du mouvement et de la multiplicité ${ }^{14} »$. Tel est manifestement le sens des mots de Baudelaire qui célébra «le transitoire, le fugitif, le contingent»; on retrouve aussi cet esprit

13. Carl E. Schorske, Thinking With History. Explorations in the Passage to Modernism (Princeton, Princeton University Press, 1998), 5. Ce passage a été traduit, mais l'italique provient de la version originale.

14. Matt. K. Matsuda, op. cit., 84. 
chez les Impressionnistes, dont l'art peignait le spectacle d'une vie quotidienne transformée par l'urbanisation. À la fin du siècle, des écrivains utiliseront de semblables images. Plusieurs souligneront l'aptitude du cerveau à accumuler un nombre incalculable d'idées ${ }^{15}$.

Cet attachement à la mémoire, qui perdurera jusqu'aux premières années $\mathrm{du} \mathrm{xx}^{\mathrm{e}}$ siècle, fut aussi associé, et de manière importante, à la montée du nationalisme et du sentiment identitaire ethnique qui assignaient une place centrale à l'histoire conçue comme vestige du passé, à la fois dans la mémoire d'un peuple et dans les traits physiques du territoire ${ }^{16}$. En Europe, cette thématique sous-tendit l'expansion territoriale et l'impérialisme. C'est pourquoi l'historienne Laura Otis a désigné la période qui va de 1870 à 1918 comme l'ère de la "mémoire organique — - cette théorie entretenant une relation intime avec le nationalisme.

S'il est important de noter que Ewald Hering fut le premier à formuler le concept de "mémoire organique » à titre d'hypothèse scientifique en 1870 , cela ne signifie pas que tous les psychologues se rallièrent à ses idées, particulièrement à la notion selon laquelle mémoire et hérédité représentent la même fonction de base de la mémoire organique. (En fait, plusieurs sommités nord-américaines la réfutèrent.) En outre, la théorie de la mémoire organique fut loin d'être la seule manière d'étudier la mémoire à la fin $\mathrm{du} \mathrm{XIx}^{\mathrm{e}}$ et au début du $\mathrm{xx}^{\mathrm{e}}$ siècle. (C'est ainsi, par exemple, que William James croyait que la capacité de se souvenir était fonction de celle de former des associations - les mêmes associations qui permettaient de former des habitudes. Plus un souvenir comportait d'associations, plus il durait. Cependant, James maintenait aussi que l'aptitude à situer le souvenir dans le passé et à l'associer comme partie de "soi» constituait le trait le plus fondamental de la mémoire — et il liait ce trait à l'hérédité.)

En fait, l'intérêt porté à la mémoire à la fin du Xix ${ }^{\mathrm{e}}$ siècle soulevait un grand nombre d'inconnues et jouait presque essentiellement comme métaphore. La mémoire était ainsi un moyen grâce auquel des universitaires de diverses disciplines exploraient des questions extrêmement controversées, comme les relations entre individu et société ou les rapports entre le mental et le physique; elle fut aussi un moyen de mener des recherches sur les mécanismes de l'hérédité ou sur le rôle de l'hérédité et du milieu dans la formation de l'individu ${ }^{17}$.

15. Ibid., 85.

16. Laura Otis, Organic Memory. History and the Body in the Late Nineteenth and Early Twentieth Centuries (Lincoln, University of Nebraska Press, 1994).

17. P. Fara et K. Patterson, loc. cit., 3 ; M. Matsuda, op. cit., 84-85. 
Reflet de cette fascination populaire et universitaire pour la mémoire, la World's Columbian Exposition de 1893 accueillit, au titre de la participation de la psychologie, un authentique laboratoire où le public pouvait vérifier l'étendue de ses capacités sensorielles et de ses facultés mentales. Première tentative de proposer des tests psychologiques à la population américaine, ce laboratoire était conçu pour montrer les méthodes utilisées par les psychologues expérimentaux pour vérifier l'étendue, la précision et la nature de ce qu'ils appelaient «certaines facultés mentales élémentaires », et pour recueillir des données en vue d'une étude plus vaste relative à l’influence, sur ces facultés, de facteurs tels l’âge, le niveau de scolarité, le sexe, la race, le milieu, le statut social et le développement physique ${ }^{18}$. La popularité de ce «kiosque» lança une mode pour les tests chez les psychologues américains qui dura peu, mais refit surface au $\mathrm{xx}^{\mathrm{e}}$ siècle. Montés sur une suite de tables, les appareillages étaient conçus pour tester l'évaluation de la longueur par les mouvements du doigt, l'évaluation du poids et du toucher, celle des surfaces par la sensation et la rapidité du mouvement, la sensibilité à la douleur; on effectuait aussi de simples tests de réaction à propos du toucher, de l'ouie et de la vision. Au cours des séances des congrès internationaux tenus conjointement lors de la foire de Chicago, de nombreuses sessions furent consacrées à la mémoire - notamment sur la question de sa conservation dans une société en proie à d'énormes changements. Les communications consacrées à la fatigue traduisirent des préoccupations semblables. Ces sessions montrèrent que le culte de l'efficience se manifestait déjà avec force à la fin du $\mathrm{xIx}^{\mathrm{e}}$ siècle et qu'il ouvrirait la voie aux notions de gestion scientifique ${ }^{19}$.

Les expériences menées à cette époque sur la vision, les temps de réaction et la mémoire n'étaient pas sans rapport avec les tâches de production qui exigeaient attention de la part de la main-d'œuvre, bien que les psychologues d'alors n'aient guère été intéressés à les utiliser dans ce contexte. Mais la psychologie allait finalement, tout comme la physiologie, servir à

18. M. Shore, "Memory in the Midst of Change», loc. cit., 71-76; J. M. Baldwin, "The Columbian Exposition : Psychology", The Nation, 26, 303-304; J. M. Baldwin, "Historical and Educational Report on Psychology ", dans Report of the Committee on Awards of the World's Columbian Commission : Special Reports upon Subjects or Groups (Washington, US Government Printing Office), House Report 1,4374, 357-404.

19 R. Badger, The Great American Fair. The World's Columbian Exposition and American Culture (Chicago, Hall, 1979), 590 ; R. H. Seager, World's Parliament of Religion. The East/West Encounter, Chicago 1893 (Bloomington, Indiana University Press, 1995); voir Proceedings of the International Congress of Education of the World's Columbian Exposition. 
modeler les individus en fonction des exigences de l'économie moderne ${ }^{20}$. L'accélération de ces orientations, après la Première Guerre mondiale, favorisa une expansion lente mais ininterrompue des sciences sociales au Canada. Dans une certaine mesure, ces idées furent importées par des universitaires américains en poste dans des universités canadiennes et par des Canadiens qui poursuivaient des études supérieures aux États-Unis. Les idées et les méthodes ainsi introduites au Canada n'y tombèrent pas d'abord en terrain fertile, car l'urbanisation et l'industrialisation y suivaient un rythme plus lent et la tradition de la philosophie morale y était encore très forte - on résistait à en détacher des disciplines telles la sociologie et la psychologie. C'est principalement pour cela que, dans plusieurs universités canadiennes, les cours de sociologie et de psychologie demeurèrent sous l'égide de la philosophie ou de l'économie politique bien après la Deuxième Guerre mondiale; cela explique aussi pourquoi plusieurs de ces universités restèrent réticentes, jusqu'aux années 1960, à adopter un système électif.

Dès la création des premières institutions d'enseignement supérieur dans les colonies britanniques d'Amérique du Nord, la philosophie de l'esprit et la morale, de même que la théodicée ou théologie naturelle, ne servirent pas seulement à inculquer aux étudiants des principes moraux religieux, mais furent aussi perçues comme des moyens de sauvegarder la tradition britannique et protestante dans des communautés séparées géographiquement de la mère patrie. Au milieu du $\mathrm{XIX}^{\mathrm{e}}$ siècle, cependant, les enseignants de l'Amérique du Nord britannique étaient confrontés à un problème : comment répondre à l'intérêt du public envers la science et la technologie - et à la nécessité de former une élite coloniale capable de développer les ressources économiques - tout en faisant en sorte que les traditions sociales et religieuses héritées de la Grande-Bretagne ne soient pas minées par le progrès. À McGill, on sentait aussi le besoin de protéger ces traditions de l'influence croissante de l'ultramontanisme ${ }^{21}$.

20. Anson Rabinbach, The Human Motor. Energy, Fatigue, and the Origins of Modernity (Berkeley, University of California Press, 1990).

21. Nous ne reprenons pas ici l'analyse détaillée du développement de la sociologie et de la recherche sociale à l'Université McGill avant la Deuxième Guerre mondiale puisqu'on retrouve cette discussion dans mon ouvrage : Marlene Shore, The Science of Social Redemption. McGill, the Chicago School, and the Origins of Social Research in Canada (Toronto, University of Toronto Press, 1987); McKillop, A Disciplined Intelligence, 28-33, 58, 95-99; E. A. Collard, "Dawson's Principalship, 1855-1893 ", dans Hugh MacLennan, dir., McGill. The Story of a University (London/Toronto, George Allen and Unwin/Nelson and Sons, 1960), 60-61. 
Les éducateurs de l'Amérique du Nord britannique s'inquiétaient des conséquences qu'aurait sur les croyances religieuses de leurs étudiants un excès d'expérimentation et de spéculation. En donnant une large place à la théodicée et à la philosophie morale, ils avaient appris à ces étudiants à concilier découvertes scientifiques et principes religieux et à associer la vie de l'esprit au commerce et aux affaires. Ils trouvèrent appui, à cette fin, dans la philosophie de l'école écossaise du Common Sense et dans la science de Bacon, qui se méfiaient toutes deux de la scolastique et n'exigeaient pas de nier l'existence de Dieu ${ }^{22}$. En plus d'inculquer des principes moraux et religieux, la philosophie morale et la théodicée étaient des disciplines intégrantes. Leur importance dans le cursus visait à dresser des barrières contre la spécialisation, dont plusieurs éducateurs craignaient qu'elle ne soit encouragée par l’insistance sur la science et sur la formation professionnelle.

Dès les années 1880 et 1890, des pressions se manifestaient déjà, au Canada, en faveur d'une formation en sciences sociales, particulièrement dans des villes comme Halifax, Montréal, Toronto et Winnipeg; le mouvement prit une nouvelle ampleur après la Première Guerre mondiale et des cours de psychologie, de sociologie et de travail social proliférèrent bientôt dans les institutions de haut savoir. Durant les années 1920 et 1930, les cours en sciences sociales se multiplièrent au Canada et firent leur entrée dans des institutions francophones, telles Laval et Montréal, où l'on s'était jusqu'alors centré sur un cursus classique en religion et en philosophie ${ }^{23}$. Les raisons à cela étaient nombreuses : d'abord le sentiment que les universités devaient être davantage à l'écoute des besoins de la société, et ensuite la foi dans le pouvoir des sciences sociales de changer les individus et de leur permettre de faire face à la société moderne. On se préoccupait aussi de l'irrationnel, espérant pouvoir le comprendre et le maîtriser. Tout cela, cependant, procédait d'une croyance dans la nécessité de l'efficience.

Ce thème de l'efficience - indifféremment appelé efficience humaine, sociale ou nationale - occupa un grand nombre d'intellectuels durant l'entre-deux-guerres. En dépit d'un halo d'utilitarisme qui n'est pas sans évoquer la "réingénierie sociale», il comportait pour certains des dimensions humanistes et même religieuses. Dans la période qui suivit la Première Guerre mondiale, on croyait généralement, au Canada, en Grande-Bretagne

22. R. A. Falconer, "Scottish Influence on Higher Education in Canada», Transactions and Proceedings of the Royal Society of Canada, 21 (May 1927) : 7-20; S. F. Cannon, «Humboldtian Science», Science and Culture (New York, Dawson and Science History Publications, 1978), 73-74.

23. Marcel Fournier, L'entrée dans la modernité : science, culture et société au Québec (Montréal, Les Éditions Saint-Martin, 1986); M. Shore, Science of Social Redemption, op cit., 32-33. 
et aux États-Unis, que la stabilité internationale reposait sur la productivité économique qui, en assurant une plus large distribution de la richesse, minimiserait les occasions de conflits. On croyait aussi qu'une telle productivité ne serait possible qu'en mobilisant une grande partie des ressources nationales pour le développement industriel, en éliminant le gaspillage et l'inefficience et en encourageant les citoyens à produire davantage. Cette conviction venait de ce que, la guerre ayant drainé d'immenses ressources humaines et matérielles, ce terrible gaspillage de vies humaines rendait nécessaire de réduire le taux de mortalité partout où cela était possible et d'augmenter l'efficience humaine ${ }^{24}$.

Dans un climat social où l'on privilégiait productivité et reconstruction, on s'intéressa naturellement au bien-être des enfants, à la prévention des troubles mentaux, aux œuvres caritatives et à l'aide sociale. Le culte de la gestion scientifique se répandit partout. Les universités adoptèrent la nouvelle mode - produire des citoyens bien formés - et elles mirent en œuvre des programmes en sciences appliquées et en formation professionnelle, technique et industrielle ${ }^{25}$. Cette mentalité stimula, elle aussi, l'expansion des sciences sociales au Canada : ses praticiens prétendirent être capables, par la recherche (et la découverte) des forces sous-jacentes à la dynamique sociale, d'orienter le développement de la société dans la bonne direction. Ils se donnèrent ainsi, pour leurs recherches, des cadres théoriques qui reposaient à la fois sur une foi aveugle dans l'observation rationnelle comme clé de la compréhension de la société et sur une conception plutôt vague de ce qu'était l'enquête scientifique.

Au Canada, c'est sans doute à McGill que cette impulsion fut la plus forte et, durant un certain temps, cette université fut à l'avant-garde du développement des sciences sociales au pays : on y créa en 1925 le premier département de sociologie autonome et, en 1930, on y entreprit un projet de recherches interdisciplinaires en sciences sociales sur le chômage et l'immigration, projet financé par la Fondation Rockefeller.

A priori, les sciences sociales convenaient bien à McGill, institution habitée d'un fort esprit utilitariste depuis les années 1850 et étroitement associée au développement de Montréal, alors capitale commerciale du Canada. Mais on n'allait vraiment s'intéresser à ces disciplines qu'après la

24. Henry Chellew, Human and Industrial Efficiency (London, University of London, 1919); Cecil Fairfield Lavell, Reconstruction and National Life (New York, Macmillan, 1919), v-viii; G. T. W. Patrick, The Psychology of Social Reconstruction (Boston/New York, Houghton Mifflin, 1920), 8-12; G. E. Patridge, The Psychology of Nations (New York, Macmillan, 1919), 290, 325.

25. H. Chellew, op. cit., 41, 68, 136. 
Première Guerre mondiale — sous l'influence de nombreux porte-parole du milieu de l'enseignement qui souhaitaient perpétuer la sensibilité aiguë aux affaires publiques provoquée par la guerre. L'argument selon lequel McGill avait le devoir de faire du Canada un pays industrialisé de premier plan aida puissamment à réunir dans ce but les ressources de toutes les disciplines : on croyait les sciences sociales capables d'aider à améliorer les conditions économiques lamentables et, ce faisant, de prévenir l'agitation sociale ${ }^{26}$.

Carl Dawson fut nommé assistant professeur de sciences sociales et directeur du programme de travail social à McGill en 1922; trois ans plus tard, il mettait sur pied un département de sociologie distinct. Formé comme ministre du culte, il avait renoncé à une vocation religieuse peu après la Première Guerre mondiale pour se consacrer aux sciences sociales. À McGill, il s'efforça d'établir la recherche comme fondement d'une compréhension de la société et d'une remise en question de l'efficience des réformes sociales. Trois facteurs avaient formé ses convictions : le milieu des baptistes des Maritimes, dans lequel il avait grandi, l'expérience de la guerre et l'éducation qu'il avait reçue. En 1914, il quitta sa région natale de l'Atlantique pour aller étudier à la faculté de théologie de l'Université de Chicago, où il se consacra surtout à ce qu'on appelait «Practical Christianity». Le service militaire interrompit ses études en 1918, mais il y revint l'année suivante pour changer radicalement d'orientation, désormais passionné par la sociologie. Cette bifurcation tenait en partie aux liens étroits qu’entretenait la faculté de théologie avec le département de sociologie - résultat du rapport historique entre sociologie et social gospel au sens large - mais résultait aussi de sa position de leader en matière de doctrine théologique radicale et libérale. Pour les modernistes de cette institution, toute étude de la société, sans excepter celle de la religion, devait se conformer aux méthodes empiriques modernes ${ }^{27}$.

À l'époque où Dawson entra à McGill, on n'était guère familier, au Canada, avec les exigences de la recherche sociale ${ }^{28}$. C'est pourquoi il passa ses premières années dans cette institution à expliquer la nature de ce type de recherche, à orienter le service social dans cette direction et à s'attaquer à la thèse, largement répandue, selon laquelle la sociologie était une discipline

26. "Changes in the Academic Side of the University", McGill Annual Report (1919-1920), 40.

27. On trouvera une analyse plus approfondie des influences intellectuelles et du milieu d'où provenait Dawson chez Marlene Shore, "Carl Dawson and the Research Ideal : The Evolution of a Canadian Sociologist», Historical Papers/Communications historiques (1985) : 45-73.

28. C. A. Dawson, «Social Research in Canada», Social Welfare, 9 (juillet 1927) : 470. 
réformiste ${ }^{29}$. Il fit ainsi partie de cette poignée d'universitaires canadiens qui, durant les années 1920, jetèrent les bases de ces travaux qu'on n'allait pas entreprendre au Canada avant les années 1930, alors que les gouvernements firent appel aux universités pour les aider à résoudre la crise économique et sociale.

Durant l'entre-deux-guerres, des sociologues formés à Chicago entreprirent des recherches sur divers lieux de la ville et formulèrent, pour leur travail, un cadre théorique et méthodologique qu'ils appelèrent écologie humaine. À McGill, Dawson affecta étudiants et collègues à l'étude de nombreuses et diverses parties de Montréal — et au-delà — et contribua aussi à la théorie de l'écologie humaine. Il entreprit une enquête systématique sur la structure de la ville et sur la manière dont elle modelait le comportement et les institutions de ses habitants. À cette époque, l'intérêt qu'on avait porté à la réforme urbaine à Montréal — de même que dans le reste du Canada — s'était dissipé et le mouvement réformiste était désuni. Tout comme il était advenu à de si nombreuses autres causes, l'enthousiasme pour la réforme urbaine mourut durant les années qui suivirent la Grande Guerre, mais la passion seule s'étiola car aux visions utopiques succéda une foi dans la gestion et l'efficience. D'un souci de l'environnement urbain, Dawson développa ensuite, fort logiquement, un projet traitant du développement de l'Ouest canadien. L'intérêt pour l'expansion et l'influence des métropoles constituait un des éléments de la théorie sociologique de l’École de Chicago. En même temps, un nouveau collègue, lui aussi formé à Chicago, se joignait à Dawson à l'Université McGill : Everett Hughes, arrivé en 1927.

Hughes se mit en frais d'étudier les communautés francophones de Montréal et de plus petites villes industrialisées, afin d'expliquer le passage du Québec de société rurale à société urbaine et industrielle. Bien qu'il ait quitté McGill en 1938 pour accepter un poste à l'Université de Chicago, plusieurs des thèmes abordés à McGill en compagnie de ses étudiants se retrouvèrent dans son livre intitulé French Canada in Transition et publié en $1943^{30}$. L'ouvrage, qui traite d'une seule petite ville (Drummondville), n'est monographique qu’en apparence. Les changements qu’il décrit, vécus par cette ville et ses habitants, sont donnés comme typiques de ce que vécut une société globale, articulée sur une culture rurale ou traditionnelle, lorsque s'imposa l'industrialisation.

29. Ibid., 470; voir M. Shore, Science of Social Redemption, op. cit., 256-261; et Fred Matthews, Quest for an American Sociology (Montréal, McGill-Queen's University Press, 1977).

30. Everett C. Hughes, French Canada in Transition (Chicago, University of Chicago Press, 1943). 
Afin d'élargir ses perspectives, Hughes avait passé un an en Allemagne à enquêter en Rhénanie où des entrepreneurs protestants avaient, au $\mathrm{XIX}^{\mathrm{e}}$ siècle, amorcé un processus d'industrialisation dans une région majoritairement catholique ${ }^{31}$. Il visait ainsi à formuler un modèle théorique de l'adaptation d'une société à l'industrialisation, un peu comme l'avait fait Robert Redfield, anthropologue de Chicago, dans son travail sur le Mexique $^{32}$. Une grande partie de French Canada in Transition et des études complémentaires menées par les étudiants et les assistants de recherche de Hughes furent consacrées à l'analyse de la paroisse rurale considérée comme principale institution formatrice de la "mentalité» canadiennefrançaise. Pour élaborer ces thèses, Hughes s'appuya sur l'œuvre du premier sociologue québécois, Léon Gérin ${ }^{33}$.

Hughes contribua aussi à introduire la recherche en sciences sociales dans les universités francophones du Québec, particulièrement à l'Université Laval où il avait fait la connaissance du père Georges-Henri Lévesque. Ce dernier, qui y avait créé un département de sciences sociales, invita Hughes à y enseigner durant un semestre en 1942-1943. Ensemble, ils mirent au point, en matière de sociologie, un programme de recherche et d'enseignement axé sur la paroisse mais qui abordait l'écologie des communautés rurales et urbaines, de même que des enquêtes sur la famille ${ }^{34}$. Ces thèmes perdurèrent durant plusieurs années à Laval sous la direction de Jean-Charles Falardeau, premier directeur laïque du département de sociologie, qui avait étudié avec Hughes à Chicago. C’est ainsi que les sciences sociales francophones, alors naissantes, adoptèrent certains traits de la sociologie de l'École de Chicago - et, par extension, de McGill. En s'attachant à la ruralité, certains sociologues francophones du Québec marchaient sur les traces de Léon Gérin et des spécialistes français de la géographie humaine et de la démographie. Les sociologues de Chicago avaient, eux aussi, adopté certains principes de cette école française. Une autre rencontre européenne avait créé des liens encore plus étroits : en 1885 et 1886, Gérin avait étudié à Paris avec Le Play et repris à son compte l'intérêt du maître pour les études axées sur la famille; or cette approche trouva écho dans l'œuvre de Hughes

31. Correspondance de l'auteure avec Everett Hughes, 18 janvier 1981.

32. E. Hughes, op. cit., 2-3.

33. Ibid., 4, 9, 10, 11.

34. Voir Everett C. Hughes, Programmes de recherches sociales pour le Québec, Cahiers de l’École de Sciences sociales, politiques et économiques de l'Université Laval, 2,4 (Québec, Les Presses de l’Université Laval, 1943). 
grâce à Robert Park qui, influencé lui-même par Le Play, avait dirigé les thèses de doctorat de Hughes et de Dawson.

C’est aussi à cette époque que furent jetées les bases du prodigieux développement de la psychologie. À la fin de la Première Guerre mondiale, on discutait beaucoup, au Canada comme ailleurs, de ce que pouvait accomplir la psychologie dans le monde de l'après-guerre. Les Américains et leurs alliés étaient allés à la guerre "to make the world safe for Democracy»; certains entendaient désormais sauver le monde "by making it psychological». Cette conviction ne fut pas étrangère à la création de cours sur les tests d'intelligence, sur la formation professionnelle et sur le développement de l'enfant.

Il est important de noter que cette expansion de la psychologie dans les universités canadiennes fut largement redevable aux activités du mouvement pour la santé mentale. Clarence Meredith Hincks, médecin, psychiatre et fondateur du Canadian National Committee for Mental Hygiene, s'intéressa beaucoup au développement de toutes les sciences sociales, qu'il estimait indispensables au bien-être de la société moderne. Son dévouement quasi religieux à la cause lui valut d'être décrit par un journaliste comme «a unique personality» — «the only generalissimo who could lead economics and the applied physical and chemical sciences, education, and preventive medicine and the social sciences in the vitally necessary struggle for a resurrected civilization ${ }^{35}$ ».

Cet espoir de voir les sciences sociales conduire, par un effort universitaire conjoint, à une «renaissance de la civilisation» résume bien la foi de ceux, fort nombreux, qui voyaient dans ces disciplines et dans la recherche interdisciplinaire la clé du relèvement social durant l'entre-deux-guerres. C'est grâce à l'influence dont jouissait Hincks que l'Université McGill obtint la subvention de la fondation Rockefeller pour entreprendre le projet de recherche sur le chômage et l'immigration mentionné plus haut. La prolifération, durant les années 1920, des projets de recherches interdisciplinaires en sciences sociales traduisait un effort de coopération visant à créer une nouvelle synthèse des connaissances susceptible de conduire à la résolution des problèmes sociaux ${ }^{36}$. À cet égard, les sciences sociales reprenaient les

35. Robert Reade, Toronto Star Weekly, sd, sp, cité dans «Hincks, Clarence Meredith», Canadian Who's Who, 2 (1936-1937) : 511-512.

36. M. Shore, The Science of Social Redemption, op. cit., chap. 6 et 7 ; voir aussi les articles de sir William Beveridge, Wesley C. Mitchell, Harold G. Moultin et Celestin Bougle, Leonard D. White, dir., dans The New Social Science (Chicago, University of Chicago Press). 
objectifs de la philosophie morale et témoignaient ainsi partiellement de leur ascendance religieuse ${ }^{37}$. On peut dire que, durant l'entre-deux-guerres, les sciences sociales s'efforçaient de créer un nouveau corpus de connaissances à la fois pour mieux comprendre et pour mieux affronter les nouvelles et difficiles conditions sociales de cette époque ${ }^{38}$.

37. Gladys Bryson, "The Emergence of the Social Sciences from Moral Philosophy ", International Journal of Ethics, 42 (avril 1932) : 304-323 ; John Higham, "The Matrix of Specialization», dans The Organization of Knowledge, 9.

38. Nos remerciements à Fernande Roy pour la soigneuse révision de la traduction française de cet article. 